\title{
Modeling Left-Turn Driving Behavior at Signalized Intersections with Mixed Traffic Conditions
}

\author{
Hong Li, ${ }^{1}$ Sujian Li, ${ }^{1}$ Haijian Li, ${ }^{2,3}$ Lingqiao Qin, ${ }^{3}$ Shi Li, ${ }^{4}$ and Zundong Zhang ${ }^{5}$ \\ ${ }^{1}$ School of Mechanical Engineering, University of Science and Technology, Beijing 100083, China \\ ${ }^{2}$ Beijing Key Laboratory of Traffic Engineering, Beijing University of Technology, Beijing 100124, China \\ ${ }^{3}$ Department of Civil and Environmental Engineering, University of Wisconsin-Madison, Madison, WI 53706, USA \\ ${ }^{4}$ MKT \& Solution Department, ZTE Corporation, Shenzhen 518057, China \\ ${ }^{5}$ School of Mechanical and Electrical Engineering, North China University of Technology, Beijing 100144, China
}

Correspondence should be addressed to Haijian Li; lihaijian@bjut.edu.cn

Received 7 January 2016; Revised 13 April 2016; Accepted 18 April 2016

Academic Editor: Luca D’Acierno

Copyright (C) 2016 Hong Li et al. This is an open access article distributed under the Creative Commons Attribution License, which permits unrestricted use, distribution, and reproduction in any medium, provided the original work is properly cited.

\begin{abstract}
In many developing countries, mixed traffic is the most common type of urban transportation; traffic of this type faces many major problems in traffic engineering, such as conflicts, inefficiency, and security issues. This paper focuses on the traffic engineering concerns on the driving behavior of left-turning vehicles caused by different degrees of pedestrian violations. The traffic characteristics of left-turning vehicles and pedestrians in the affected region at a signalized intersection were analyzed and a cellularautomata-based "following-conflict" driving behavior model that mainly addresses four basic behavior modes was proposed to study the conflict and behavior mechanisms of left-turning vehicles by mathematic methodologies. Four basic driving behavior modes were reproduced in computer simulations, and a logit model of the behavior mode choice was also developed to analyze the relative share of each behavior mode. Finally, the microscopic characteristics of driving behaviors and the macroscopic parameters of traffic flow in the affected region were all determined. These data are important reference for geometry and capacity design for signalized intersections. The simulation results show that the proposed models are valid and can be used to represent the behavior of left-turning vehicles in the case of conflicts with illegally crossing pedestrians. These results will have potential applications on improving traffic safety and traffic capacity at signalized intersections with mixed traffic conditions.
\end{abstract}

\section{Introduction}

Over the past two decades, the study of driving behavior, which is closely related to the daily traffic of individuals, has become a hotly pursued research topic. Driving behaviors are strongly related to the safety of traffic participants. In the field of driving behavior, many researchers have devoted themselves to modeling driving behavior, analyzing conflict mechanisms, and improving traffic safety. As early as 1985, Abishai studied driver behavior at unsignalized urban intersections [1]. That paper strove to investigate whether stop signs differ from yield signs in terms of their associated accident rates and whether an increase in the level of traffic control is necessarily beneficial to traffic safety at all hazardous urban intersections. Later, in 2005, Bonsall et al. and Björklund and Åberg studied safety-related driving behavior and driver behavior at intersections, respectively [2,3]. Bonsall et al. [2] identified the key parameters of traffic simulation models and concluded that it is better to use values that are realistic but unsafe than values that are safe but unrealistic. Björklund and Åberg [3] studied driver behaviors at intersections with different traffic rules and showed that drivers' reported behaviors varied among different intersections. Recently, an increasing number of researchers have shown interest in normal driving behavior models [4-9], driving simulators [10-13], car-topedestrian incidents [14], and even angry and aggressive driving behavior [15-17].

Yan and Radwan [4] studied driver behaviors at signalized intersections. To identify the changes in driving behaviors that could be clearly associated with restricted sight distances of drivers, they developed linear regression and logistic regression models to estimate gap-acceptance parameters. They found that sight obstruction may contribute to significant increases in the critical gap and follow-up time and 
that left-turning or U-turning drivers with restricted sight distances were more likely to accept smaller gaps. Zhang et al. [5] evaluated the effects of in-vehicle warning information on drivers' decelerating and accelerating behavior when approaching an intersection near an arch-shaped bridge and found that dynamic information appears to be more effective than static information in allowing drivers to avoid dangerous driving situations. Moreover, Elhenawy et al. [7] studied a model of stop/run driver behavior at signalized intersections considering roadway surface conditions. Bar-Gera et al. [8] focused on crossings during the yellow-light phase and the first $1.5 \mathrm{~s}$ of the red-light phase, and they found that, at most of the intersections under study, the frequencies of entrance times after yellow-light onset were reasonably stable during the beginning of the yellow-light phase. In 2015, Lin et al. [9] analyzed conflicts between right-turning vehicles and through-traveling nonmotorized vehicles. They developed a micro driving force model and demonstrated that strict priority crossing behavior is a special case of the proposed driving force model. The proposed model can be used to predict the average crossing speed of right-turning vehicles.

Driving-simulator-based methods are also increasingly being used to study driving behaviors. Muhrer and Vollrath [10] directed their efforts toward better understanding the causes of driver errors in the context of rear-end crashes in a simulated driving task. Riccardo et al. [11] proposed a comparative analysis of random utility models and fuzzy logic models for representing gap-acceptance behavior using data from driving simulator tests. In 2015, Matsumoto and Peng [13] also proposed a method of obtaining information based on vehicle operating conditions and traffic signal status for decision-making regarding whether to pass through a signalized intersection ahead using a 3D driving simulator. They found that the proposed information is effective for reducing $\mathrm{CO}_{2}$ emissions. To gain an in-depth understanding of how and why safety-critical situations occur, Habibovic et al. [14] analyzed causation in video recordings of car-pedestrian incidents captured by onboard cameras and demonstrated two major patterns of causation: the drivers often failed to recognize the presence of conflicting pedestrians, and the pedestrians often behaved in unexpected ways. Moreover, research on angry and aggressive driving behaviors has also been performed. Kaysi and Abbany [15] investigated the aggressive behavior of minor street vehicles at priority-unsignalized intersections and modeled aggressive driver behavior at unsignalized intersections. They revealed that age, car performance, and average speed are the major determinants of aggressive behavior.

Recently, cellular-automata-based driving behavior models [18-20] have been studied in depth. Hu et al. [19] used a typical KKW (Kerner-Klenov-Wolf) cellular-automata (CA) model to investigate the formation mechanism of the illegal lane-changing behavior of nonmotorized vehicles. Chen et al. [20] proposed a cellular-automata-based traffic model to describe mixed traffic.

Mixed traffic conditions are a very common phenomenon in urban transportation systems. Mixed traffic exhibits complex characteristics. Mixed traffic flow is a very common scenario in many cities in developing countries, especially
China and India. Many publications in the literature have addressed characteristics, models, and theories of mixed traffic flow. Liang et al. and Prasetijo et al. [21-23] focused on the capacity of various intersections under mixed traffic conditions. As mentioned above, $\mathrm{Hu}$ et al. [19] and Chen et al. [20] also studied the mixed traffic modeling problem. Moreover, Marisamynathan and Vedagiri [24] developed a pedestrian delay model for crosswalks at signalized intersections under mixed traffic conditions, and they verified the validity of the proposed model using field data from India. Anjana and Anjaneyulu [25] studied the causative factors of crashes at signalized intersections with mixed traffic and developed models for predicting crash frequency and severity. One of their findings was that dedicated left-turn lanes and countdown timers are beneficial for improving the safety of signalized intersections.

Car-following behavior is more complicated under mixed traffic conditions than it is in homogeneous traffic. Traditional car-following models are concerned only with traffic flows consisting of motorized vehicles of a single type. In 1974, Wiedemann [26] proposed the use of a behavioral threshold to distinguish different following states and established a behavioral threshold model based on this approach. To date, Wiedemann's following model remains one of the most accurate models for use in computer traffic simulations. The car-following model in the microscopic traffic simulation software package VISSIM is also based on Wiedemann's following model [26-28]. For single-vehicle traffic flow, VISSIM can successfully reflect and reproduce actual traffic conditions. However, in developing countries, urban mixed traffic is the most common form of transportation; traffic of this type faces many major problems, such as conflicts, inefficiency, and security issues. Therefore, traditional vehicle flow models cannot truly reflect the driving behavior of motorized vehicles under mixed traffic conditions. Conflicts between motorized vehicles and pedestrians and between motorized vehicles and nonmotorized vehicles have become important issues in the modeling of mixed traffic at many intersections.

Most of the literature on driving behavior has focused either on single-motorized-vehicle traffic flow or on aspects related to mixed traffic rather than addressing all of these factors (driving behaviors, CA models, and mixed traffic conditions) simultaneously. These studies have not considered the impact of vehicle-pedestrian conflicts resulting from different degrees of pedestrian violations under mixed traffic conditions. Accordingly, this paper investigates the effects of pedestrian violations on vehicles, especially left-turning vehicles. The ability to control violations of this kind would have important significance in improving intersection capacity and relieving traffic pressure.

\section{Mixed Traffic Characteristics and Vehicle- Pedestrian Conflicts}

Vehicle-pedestrian conflicts represent the majority of problems encountered at most intersections in China. These conflicts cause observable decreases in capacity, greatly increase the number of traffic accidents, and considerably reduce 
transportation benefits at intersections. In several large cities, the problem of pedestrian violations has become increasingly serious, and it places severe constraints on the driving behaviors of motorized vehicles, especially left-turning vehicles. In the context of this phenomenon, this paper focuses on the impact on driving behavior of left-turning vehicles caused by different degrees of pedestrian violations.

2.1. Characteristics of Vehicle Traffic. At urban intersections, the traffic flows on intersecting roads interact through merging, steering, and diverging. Because of their different directions of travel, when nonmotorized vehicles, motorized vehicles, and pedestrians reach an intersection in accordance with their different probability distributions, the traffic flows first begin to diverge and then to merge, causing travel speeds to be reduced and disrupting normal traffic flow. The larger the number of directions in which the traffic flows diverge is, the more serious the interference conflicts will be. At an intersection, the left-turning and through-traveling traffic flows proceeding in different directions form a point of conflict. Consequently, vehicles (especially left-turning vehicles) proceeding in different directions will conflict with nonmotorized vehicles and pedestrians during the same traffic-light phase. To avoid collisions, these vehicles must travel slowly, decelerate, or even stop.

2.2. Characteristics of Pedestrian Traffic. Pedestrian traffic exhibits several typical characteristics: a single pedestrian occupies a small space, has a fast start-up speed, can execute abrupt turns, and is always able to perform an illegal crossing. Pedestrians tend to form into groups when entering an intersection. The populations originating from a mixed traffic flow are typically concentrated in the left and right corners (with respect to the direction of left-turning vehicles) and at the crosswalks at an intersection; these locations constitute several key nodes of mixed traffic flow in a road network. Pedestrians' intersection-crossing behavior can be predominantly characterized in terms of factors such as crossing speed, spacing requirements, waiting time, and step size. In this paper, we focus on studying the traffic characteristics of pedestrians when crossing a street under signal control; therefore, the two main aspects of this type of pedestrian traffic that will be defined and discussed are the tolerable waiting time and the crossing speed.

(i) Tolerable Waiting Time. The tolerable waiting time is the maximum acceptable time for which pedestrians will wait to cross a signalized intersection and is mainly related to the volume of traffic on the road, the vehicle speed, the road width, the time required to cross, and other traffic conditions. For example, the maximum tolerable waiting time of pedestrians in the UK ranges from 45 to 60 seconds and is only 28 seconds in Japan but may be as long as 70 to 90 seconds in China, according to various surveys. The tolerable waiting times of pedestrians given different road conditions can be determined through on-site investigations at local intersections.

(ii) Pedestrian Crossing Speed. When crossing a street, the walking speed of pedestrians is strongly influenced by the crossing signal and by the crowd crossing in the opposite direction. In addition, the crossing speed of illegally crossing pedestrians is also influenced by the speed and number of vehicles involved in the pedestrian-vehicle conflict at that time. Statistics indicate that the average pedestrian crossing speed in China is $1 \sim 1.35 \mathrm{~m} / \mathrm{s}$.

2.3. Vehicle-Pedestrian Conflict Analysis. As early as the 1970s, Campbell and King [29] studied the application of the traffic conflicts technique. The traffic conflicts technique is a systematic method of observing traffic at an intersection to determine the existing potential for traffic accidents. In [29], the authors defined a traffic conflict as any potential accident situation, which may be identified by observing (i) evasive actions taken by drivers to avoid a collision and (ii) traffic violations. To enable the flexible and effective use of the traffic conflicts technique under the unique traffic conditions that prevail in China, Liu and Duan [30] described and defined the phenomenon of traffic conflicts at intersections. They observed that at an intersection, two or more road users will approach each other at the same time and spatial location. Therefore, if one of these users fails to take measures to change his or her normal traffic behavior, such as changing directions, reducing speed, or stopping and waiting, then only if the others take suitable action will danger be averted; if not, these users will be in a collision situation.

Conflicts between a vehicle and other vehicles or pedestrians at signalized intersections may be of the crossing type, the merging type, or the diverging type, and, of these, crossing-type conflicts always possess a more severe accident potential. Generally, crossing-type conflicts involving vehicles and pedestrians at a signalized intersection can be classified into three types: (i) crossing conflicts between leftturning vehicles and straight-traveling vehicles; (ii) crossing conflicts between left-turning vehicles and straight-traveling pedestrians; and (iii) crossing conflicts between right-turning vehicles and straight-traveling pedestrians. Among these three kinds of crossing conflicts, the conflict mechanism and behavior model for conflicts between left-turning vehicles and straight-traveling pedestrians, especially illegally crossing pedestrians, will be the most severe and complicated; therefore, this paper places the greatest emphasis on conflicts of this type.

(1) Conflicts between Left-Turning Vehicles and Pedestrians. For an independent left-turn phase at an intersection, when the left-turn signal light turns green, the left-turning traffic begins traveling and may face conflicts with illegally crossing pedestrians traveling in the vertical direction. The affected regions and conflict points in a left-turning vehiclepedestrian conflict scenario are illustrated in Figure 1.

(2) Analysis of Driving Behavior under Man-Machine Conflict Conditions. Based on the work of Schroeder [31], this paper defines four modes of behavior of the left-turning vehicles that are passing through the vehicle-pedestrian conflict region (as shown in Figure 1): "crossing at uniform speed," "crossing while decelerating," "crossing slowly," and "braking and stopping." 


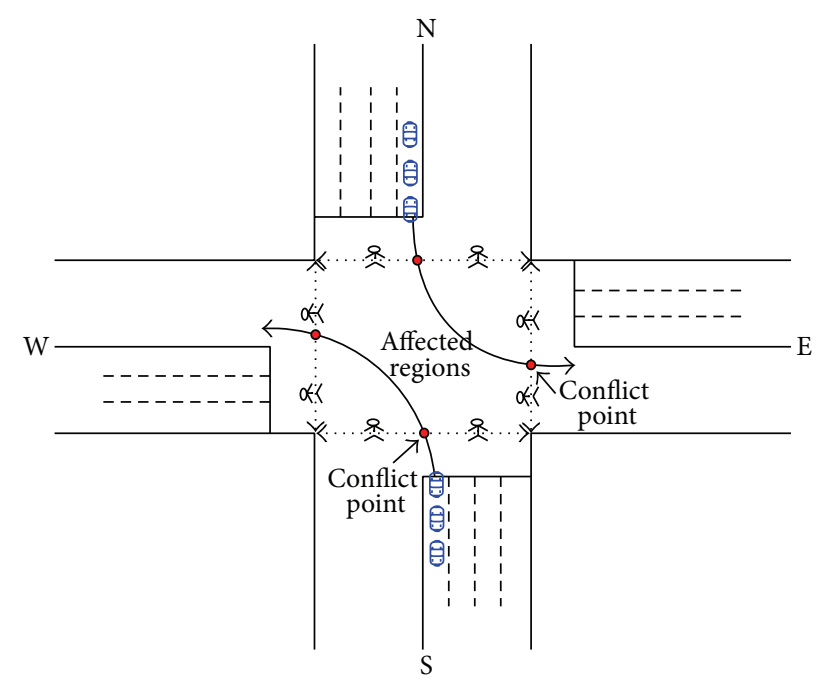

FIGURE 1: Affected regions and conflict points in a left-turning vehicle-pedestrian conflict scenario.

Mode 1 (crossing at uniform speed). When no pedestrian violation phenomenon is occurring at an intersection, only the vehicles ahead affect driver behavior, and the left-turning vehicles cross through the affected region at the intersection in the normal car-following mode.

Mode 2 (crossing while decelerating). When there are illegally crossing pedestrians at an intersection, the physiology and psychology of the drivers of left-turning vehicles will be impacted. To ensure that there are no direct conflicts with pedestrians, left-turning vehicles will approach the intersection in the normal car-following mode and will then enter a state of deceleration before crossing the intersection.

Mode 3 (crossing slowly). When a larger number of illegally crossing pedestrians are present in the affected region, the vehicle-pedestrian conflict will severely affect the normal behaviors of motorized vehicles, and the left-turning vehicles will cross the affected region at a slower speed to avoid direct conflicts with pedestrians.

Mode 4 (braking and stopping). When the distance between a vehicle and the vehicle ahead is less than the minimum desired following distance or the vehicle is in direct conflict with pedestrians, the driver will take the actions of braking and then stopping to avoid a potential crash. After conflict avoidance, the vehicle will resume motion and continue driving.

To simultaneously consider the two factors of the vehicles ahead and vehicle-pedestrian conflicts, this paper establishes a left-turn driving behavior model for vehicles based on CA model to study the behavior mechanisms of left-turning vehicles at signalized intersections.

\section{Driving Behavior and Relative Share Models}

3.1. Theoretical Basis and Motivation. In the 1950s, Neumann proposed the concept of cellular automata [32]. A CA model is a discrete space-time model of local dynamics and a common technique used in complex systems research. This approach is particularly suitable for simulation studies of the temporal dynamics of complex spatial systems, and it has been widely applied and developed for use in the study of traffic and transportation systems. Wolfram's CA model (a onedimensional transport model) serves as a clear demonstration of the successful application of CA theory in the study of traffic [33]. The NS model proposed by Nagel and Schreckenberg in 1992 is a CA model of traffic flow in the truest sense [34]. Many other investigators have made subsequent improvements based on the NS model afterwards, and much of the research on the modeling of microscopic traffic flow has focused on this model. The NS traffic flow model is simple and especially easy to implement on a computer; moreover, it is capable of reproducing a variety of complex traffic phenomena and accurately reflects the characteristics of traffic flow.

By studying the changes in cellular state that occur during a simulation based on a CA model, we can obtain not only the velocity, location, speed, headway, and other parameters of each vehicle at any moment in time to describe the microscopic characteristics of the traffic flow but also the average speed, density, vehicle counts, and other parameters to describe the macroscopic traffic flow characteristics. Furthermore, the traffic flow modeling of lanes, multilane roads, road networks, and motorized and nonmotorized vehicles can also be achieved. Therefore, by considering vehicle-pedestrian conflicts on the basis of the NS model, this paper establishes a "following-conflict" driving behavior model that can reflect the real characteristics of left-turning vehicle-pedestrian conflicts at signalized intersections.

3.2. "Following-Conflict" Driving Behavior Model Based on $C A$. The purpose of this paper is to study the mechanisms governing the behavior of left-turning vehicles in the affected region at a signalized intersection and to simulate the changes in the speed, flow, and other parameters of left-turning vehicles that are induced by different degrees of conflict with illegally crossing pedestrians. Accordingly, a cellular-automatabased "following-conflict" driving behavior model is established to simulate the driving behavior of left-turning vehicles and reflect the state transitions of the various driving behaviors of such vehicles at signalized intersections. This model transforms the actual driving behaviors of traffic engineering into specific mathematic expressions which are simulated by computer programs. A diagram of the finite-state machine representing the driving behavior of left-turning vehicles in the affected region is shown in Figure 2.

The finite-state machine is divided into two main procedures: one is the addition of new vehicles to the cell area, which is determined by the grid density and the proportion of vehicles, and the other is the status update of the existing vehicles and the newly added vehicles, which converts the normal car-following status of each vehicle into one of a variety of driving behavior modes in the affected region.

(1) Definitions of Car-Following Behaviors between Vehicles. With regard to car-following behaviors between vehicles, this 


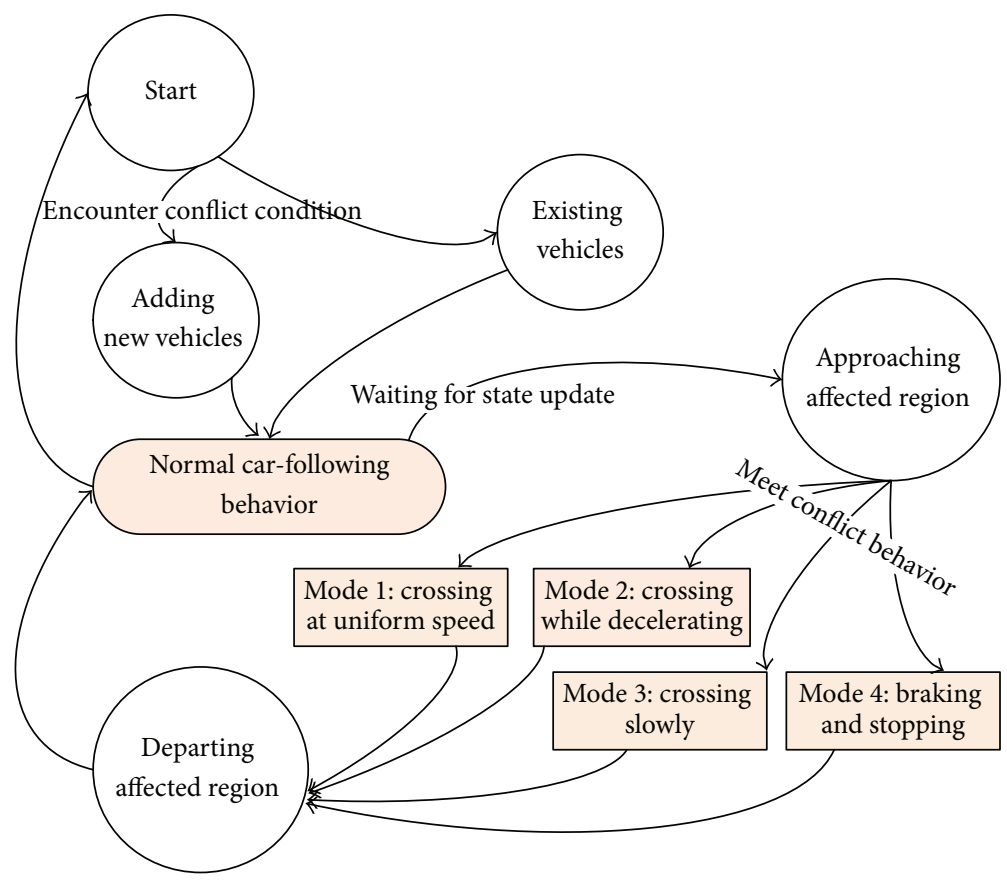

FIGURE 2: Finite-state machine representing the driving behavior of left-turning vehicles in the affected region.

paper adopts a classical following rule, which includes velocity updating, random velocity moderation, location updating, a minimum safety distance, and other parameters. Accordingly, the car-following behaviors are defined as follows:

(i) acceleration: $v_{n}(t+1)=\min \left(v_{n}(t)+1, v_{\max }\right)$,

(ii) deceleration: $v_{n}(t+1)=\min \left(v_{n}(t), d_{n}\right)$,

(iii) random velocity moderation (with probability $q$ ): $v_{n}(t+1)=\max \left(v_{n}(t)-1,0\right)$,

(iv) location update: $x_{n}(t+1)=x_{n}(t)+v_{n}(t+1)$,

where $v_{n}(t)$ denotes the speed of vehicle $n$ at time $t ; d_{n}$ is the number of empty cells between the vehicle $n$ and its preceding vehicle $n+1$ and is defined as $d_{n}=x_{n+1}-x_{n}-l_{n+1}$, where $l_{n+1}$ denotes the length of vehicle $n+1$, that is, the number of cells occupied by vehicle $n+1 ; q$ is the probability of random velocity moderation; and $v_{\max }$ is the maximum vehicle speed permitted at a signalized intersection.

(2) Models of Driving Behavior in the Affected Region. According to the different vehicle situations and the car-following behaviors between vehicles as described above, the four possible modes of left-turning vehicle behaviors in the case of conflicts with pedestrians in the affected region at a signalized intersection are modeled as follows.

Mode 1 (crossing at uniform speed). The following state of a vehicle in this mode in the affected region is as follows:

Speed update: $v_{n}(t+1)=v_{n}^{(1)}(t)$,

Location update: $x_{n}(t+1)=x_{n}(t)+v_{n}^{(1)}(t)$, where $v_{n}^{(1)}(t)$ is the invariant speed of Mode 1 in the following state.

Mode 2 (crossing while decelerating). In this mode, several potential conflicts may arise that may affect the traveling or normal car-following behaviors of left-turning vehicles. As it approaches the affected region at an intersection, a vehicle in this mode enters a decelerating state to ensure that it will reach a lower speed before it encounters a potential conflict point. Therefore, the speed $v_{n}(t+1)$ at the next instant of time will be randomly distributed on the interval $\left[v_{\text {low }}^{(2)}, v_{\text {top }}^{(2)}\right]$, yielding the following:

$$
\begin{aligned}
\text { Speed update: } v_{n}(t+1) & =v_{\text {low }}^{(2)}+\varepsilon^{(2)}, \\
\text { Location update: } x_{n}(t+1) & =x_{n}(t)+v_{n}(t+1),
\end{aligned}
$$

where $\varepsilon^{(2)}$ is a parameter of Mode 2, which is a random variable on $\left[0, \Delta v^{(2)}\right]$, where $\Delta v^{(2)}=v_{\text {top }}^{(2)}-v_{\text {low }}^{(2)} ; v_{\text {low }}^{(2)}$ is the minimum speed possible during the deceleration process; and $v_{\text {top }}^{(2)}$ is the maximum speed possible during the deceleration process.

Mode 3 (crossing slowly). In this mode, a left-turning vehicle travels at a slower speed to avoid potential conflicts with illegally crossing pedestrians in the affected region when passing through the intersection. Consequently, it can be assumed that the speed at the next instant of time, $v_{n}(t+1)$, will be randomly distributed on $\left[v_{\text {low }}^{(3)}, v_{\text {top }}^{(3)}\right]$ :

Speed update: $v_{n}(t+1)=v_{\text {low }}^{(3)}+\varepsilon^{(3)}$,

Location update: $x_{n}(t+1)=x_{n}(t)+v_{n}(t+1)$, 
where $\varepsilon^{(3)}$ is a parameter of mode 3 , which is a random variable on $\left[0, \Delta v^{(3)}\right]$, where $\Delta v^{(3)}=v_{\text {top }}^{(3)}-v_{\text {low }}^{(3)} ; v_{\text {low }}^{(3)}$ is the minimum speed possible during the slow passing process; and $v_{\text {top }}^{(3)}$ is the maximum speed possible during the slow passing process.

Mode 4 (braking and stopping). A left-turning vehicle may face a direct conflict with pedestrians, such that it must brake and stop immediately to avoid a crash. In this case, the model for the updating of the driving behavior state is as follows:

$$
\begin{aligned}
& \text { Speed update: } v_{n}(t+1)=0 \text {, } \\
& \text { Location update: } x_{n}(t+1)=x_{n}(t)+0=x_{n}(t) \text {. }
\end{aligned}
$$

Once the conflict disappears, the vehicle resumes motion and continues to travel in normal car-following behavior mode with the vehicles ahead.

(3) Relative Share Model of Driving Behavior Choices. Depending on the actual situation, there are four possible modes of the driving behavior of left-turning vehicles in the affected region; here, we analyze the relative share of each mode based on a logit model. The logit-model-based method of relative share analysis makes it possible to consider a variety of external factors. Furthermore, this method is more representative than other methods for this purpose and can also yield consistent parameter estimates for each parameter set. Thus, the logit-model-based method of estimating the relative shares of the different behavior modes yields results that are closer to the actual situation.

In this paper, the driving behavior of left-turning vehicles at a signalized intersection is the typical scenario of interest. Survey results indicate that, at an intersection with a separate set of left-turn signal lights, the speed of the left-turning vehicles is related not only to the geographical environment of the intersection but also to various characteristics of the motorized vehicles and pedestrians that are present. The most important and typical time factors, namely, the time required for a vehicle to cross the intersection, the time required for an illegally crossing pedestrian to cross the affected region, and the time required for a pedestrian to cross the crosswalk, are used to analyze the relative shares of different behavior modes.

(i) The Time Required for a Vehicle to Cross the Intersection $\left(t_{1}\right)$. When a left-turning vehicle is on the right-of-way, $t_{1}$ represents the time that the vehicle will take to travel the distance between the stop line and outer edge of the second vertical crosswalk. $t_{1}$ will differ for different driving behavior modes. A shorter $t_{1}$ will lead to less conflict with pedestrians, allowing more vehicles to pass through the intersection and thus corresponding to a greater value of the weight parameter used in the logit model.

(ii) The Time Required for an Illegally Crossing Pedestrian to Cross the Affected Region $\left(t_{2}\right) . t_{2}$ is the time interval that is required for an illegally crossing pedestrian to cross the affected region when left-turning vehicles are on the rightof-way. In a realistic situation, with different driving behavior modes for the vehicles, a shorter illegal-pedestrian-crossing time interval will result in a weaker impact on the motorized vehicles at the intersection, allowing more vehicles to pass through and thus corresponding to a greater value of the weight parameter used in the logit model.

(iii) The Time Required for a Pedestrian to Cross the Crosswalk $\left(t_{3}\right), t_{3}$ represents the time required for a pedestrian to cross on a straight crosswalk. In the realistic case of different driving behavior modes of left-turning vehicles, a longer $t_{3}$ results in a lower probability of violation (avoiding the possibility that pedestrians will become isolated in the middle of the crosswalk by vehicles); in turn, the impact on the motorized vehicles is weaker, and the value of the weight parameter used in the logit model is greater.

By adopting the time factors analyzed above as the main features of the logit model, we can construct a generalized time function with the following specific form:

$$
V_{i}=\theta_{1} t_{i 1}+\theta_{2} t_{i 2}+\theta_{3} t_{i 3}+\varepsilon_{i}
$$

where $V_{i}$ is the generalized time function for driving behavior mode $i(i=1,2,3$ or 4$) ; \theta_{1}, \theta_{2}$, and $\theta_{3}$ are the weight coefficients corresponding to time factors $t_{1}, t_{2}$, and $t_{3}$, respectively; $t_{i 1}, t_{i 2}$, and $t_{i 3}$ are the values of the corresponding time factors $t_{1}, t_{2}$, and $t_{3}$, respectively, of mode $i$; and $\varepsilon_{i}$ is the random error of mode $i$, where it is assumed that the utility functions of the random errors $\varepsilon_{i}(i=1,2,3$, or 4$)$ are independent and follow a Gumbel distribution.

Then, the relative share of each mode $i$ in the choice of driving behaviors can be calculated based on the logit model approach using (5). According to random utility theory, the probability $P_{i}$ that the $i$ th driving behavior mode will be selected is

$$
P_{i}=\frac{\exp \left(V_{i}\right)}{\sum_{i=1}^{4} \exp \left(V_{i}\right)}
$$

\section{Numerical and Field Experiments}

4.1. Driving Behavior Analysis Based on the "FollowingConflict" Model. To more intuitively describe the influence of illegally crossing pedestrians on the driving behavior of left-turning vehicles, we simulated four modes of left-turning vehicle driving behavior and then analyzed the impact on the flow, speed, and other parameters of left-turning vehicles experiencing different degrees of conflict with illegally crossing pedestrians at a signalized intersection.

For the experiment, the Mudanyuan intersection (牡丹 园交叉口) (one of many signalized intersections with an independent signal phase for left-turning vehicle in Beijing, China) was selected as the environment for the simulation of vehicle-pedestrian conflicts to verify the model and method proposed in this paper. For consistency with the geometrical characteristics and actual traffic flow state of the Mudanyuan intersection, we initialized the length of one cell as $1.5 \mathrm{~m}$ and the width of one cellular space as one vehicle lane. Moreover, one standard vehicle was considered to occupy 4 cells, the length of the simulation area was defined as 800 cells, the leftturning area was defined to occupy between 50 and 80 cells, 


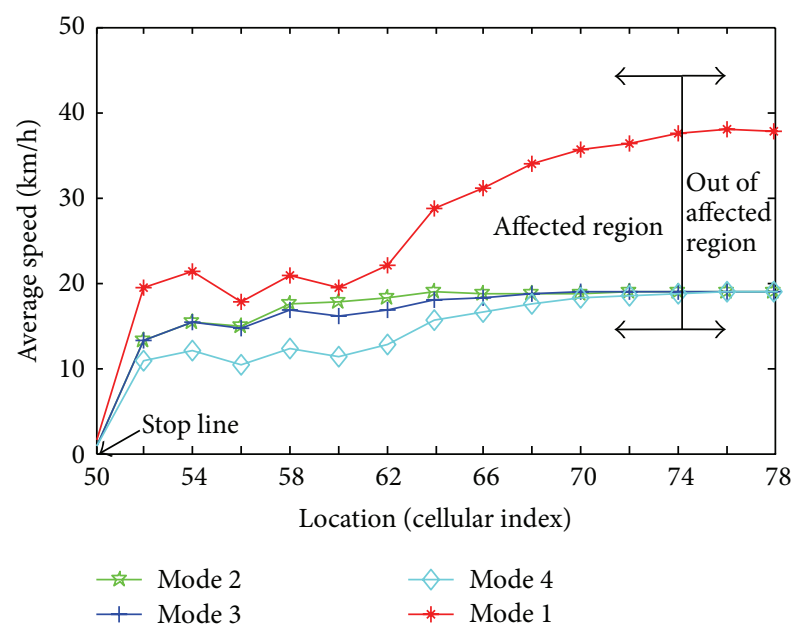

Figure 3: Average speed trend in each mode in the left-turn area.

the maximum permitted speed for vehicles in the affected region was defined as 8 cells per 1-second time step, and the total length of the simulation time was 4500 seconds.

In Figure 3, the location as represented by the cellular index is shown on the horizontal axis and the vertical axis shows the average speeds of vehicles in the simulation as they passed by these cell locations. The spatial interval between the 50th and 80th cells corresponds to the leftturn area in the simulation experiment. It is evident that as the severity of the vehicle-pedestrian conflict increases, from Modes 1-4 of left-turn driving behavior, the average speed of the left-turning vehicles decreases. However, because of the continuous influence of following behavior, in each mode, the average speeds at different locations exhibit an increasing trend with an increase in the cellular index.

To reveal the driving behavior mechanism of each mode, Figure 4 presents the space-time curves of each simulated vehicle in the affected region of the signalized intersection for each behavior mode. In Figure 4, the slope of any arbitrary point on each vehicle's space-time curve represents the vehicle's instantaneous velocity. As shown in Figure 4, when no pedestrian violations exist or when such violations play a minor role in affecting traffic (Modes 1 and 2), the space-time curve of each vehicle is steep; that is, a high instantaneous velocity is observed in the simulated region. As the degree of impact of pedestrian violations grows (Modes 3 and 4), the space-time curves gradually become more shallow, resulting in lower instantaneous velocities. Consequently, the delays of the left-turning vehicles increase. Moreover, for a simulation of a single left-turn lane, a straight lateral line passing through these space-time curves at a fixed location will generate the headway time distribution for each left-turn driving behavior mode; similarly, a straight vertical line will generate the headway space distribution.

For analysis of the macroscopic characteristics of traffic flow in the affected region, Figure 5 presents the average flowdensity curve for each behavior mode of left-turning vehicles as derived from our simulation results. Because the density of left-turning vehicles in the affected region is small, Figure 5 shows only a small region with low flow and low density compared with a classical flow-density curve. However, Figure 5 still exhibits the natural relationship between low flow and low density, and as the traffic density increases, the average flow also increases for each behavior mode. A comparison of the different behavior modes reveals that as the degree of vehicle-pedestrian conflict grows (Modes 1-4), the average flow of the left-turning vehicles decreases. Meanwhile, Figures 6(a) and 6(b) present additional microparameters of traffic flow, namely, the average flow and average speed in every $600 \mathrm{~s}$ time interval for each behavior mode. As shown in Figure 6, as the degree of vehicle-pedestrian conflict grows, the average flow and average speed in a fixed time interval both decrease. The simulation results of the cellularautomata-based model proposed in this paper agree well with the characteristics of the real situation.

4.2. Relative Share Analysis. In addition to its use as a simulation object for the analysis of the driving behavior modes of left-turning vehicles at signalized intersections with mixed traffic condition, the Mudanyuan intersection was also used to analyze the relative share of each behavior mode. This intersection is a typical four-phase signalized intersection whose pedestrian and bicycle phases are identical to those for the motorized vehicles that are traveling straight through the intersection in the same direction. The Mudanyuan intersection connects two main arterials, the Beitucheng West Road (北土城西路) and the Huayuan East Road (花园 东路), and serves to link the 3rd and 4th ring expressways in Beijing. Moreover, it is also close to a metro station on Line 10 (Mudanyuan Metro Station). Consequently, the Mudanyuan intersection always has a heavy flow of mixed traffic, which results in violent and continuous traffic conflicts between motorized vehicles and nonmotorized vehicles or pedestrians, especially left-turning vehicles and illegally crossing pedestrians. Therefore, this intersection is a typical and practical intersection at which to research left-turning vehicle-pedestrian conflicts under mixed traffic conditions.

Before the logit model could be used, it was necessary to first obtain the time factors $\left(t_{1}, t_{2}, t_{3}\right)$ for the Mudanyuan intersection. Fortunately, these parameters could be calculated from field investigation data collected in a large number of field surveys. The statistical results for these three time factors at the Mudanyuan intersection are shown in Table 1.

Second, it was necessary to determine the weight coefficients for the time factors $\left(\theta_{1}, \theta_{2}, \theta_{3}\right)$. Using the data in Table 1 and (5), the weight coefficients were determined using the Biogeme software package [35] and the results are shown in Table 2.

Third, using (5) and (6), the probabilities of the various behavior modes, where $P_{i}$ represents the probability of the $i$ th driving behavior mode, were determined to be $P_{1}=0.5, P_{2}=$ $0.1, P_{3}=0.3$, and $P_{4}=0.1$.

To test the validity and practicability of the logit model proposed in this paper, additional simulation experiments were performed using the probabilities $P_{i}$ derived from the logit model and the field data. Table 3 compares the statistical results for the left-turning traffic flow obtained through 

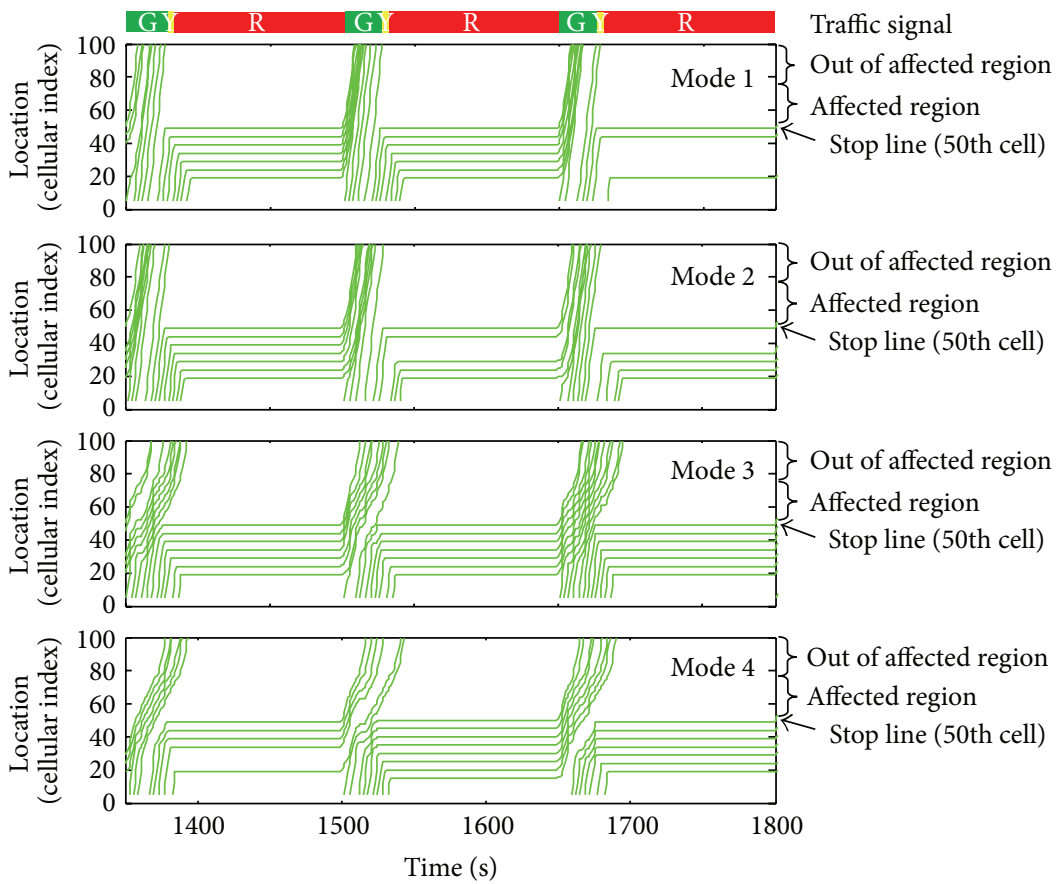

FIGURE 4: Space-time curves for left-turning vehicles in the affected region for each behavior mode.

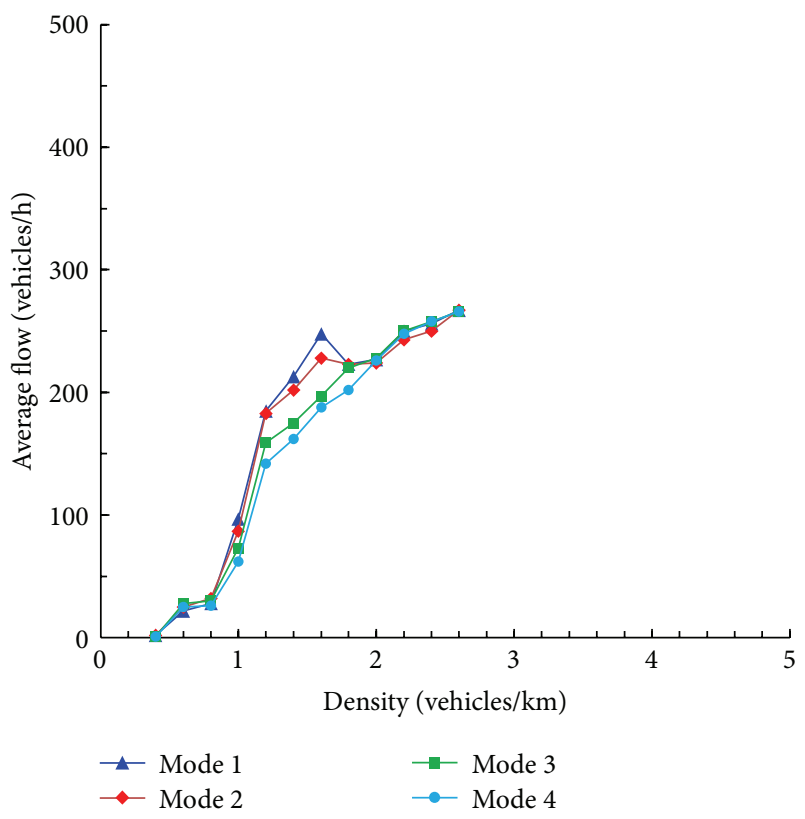

FIGURE 5: Average flow-density curve for each behavior mode of leftturning vehicles.

simulations and from the field data. Moreover, Figure 7 presents the detailed statistical results for the left-turning traffic flow in each fixed time interval of $600 \mathrm{~s}$ from 7:40 AM to 8:30 AM. Table 3 and Figure 7 illustrate that more severe vehicle-pedestrian conflicts lead to smaller traffic flows, in turn causing more traffic delays and safety risks at a signalized
TABLE 1: Statistical results for the three time factors at the Mudanyuan intersection.

\begin{tabular}{lccc}
\hline $\begin{array}{l}\text { Left-turning vehicle driving } \\
\text { behavior modes }\end{array}$ & $t_{1}(\mathrm{~s})$ & $t_{2}(\mathrm{~s})$ & $t_{3}(\mathrm{~s})$ \\
\hline Mode 1 & 6.21 & 7.94 & 21.28 \\
Mode 2 & 7.91 & 8.37 & 21.92 \\
Mode 3 & 8.57 & 9.43 & 22.96 \\
Mode 4 & 10.38 & 11.44 & 24.96
\end{tabular}

TABLE 2: Weight coefficients for the logit model.

\begin{tabular}{lccc}
\hline Weight coefficients & $\theta_{1}$ & $\theta_{2}$ & $\theta_{3}$ \\
Values & 0.498 & 0.619 & 0.862 \\
\hline
\end{tabular}

intersection with mixed traffic conditions. Therefore, in engineering, to reduce these conflicts will be an essential concern for intersection planning and design. Moreover, the consistency between the simulation results and the field data also demonstrates the validity and accuracy of the logit relative share model, the cellular-automata-based "followingconflict" model, and the corresponding simulation methods proposed in this paper.

\section{Conclusions}

This paper analyzed the characteristics of conflict between left-turning vehicles and illegally crossing pedestrians at signalized intersection, which is a common phenomenon under 


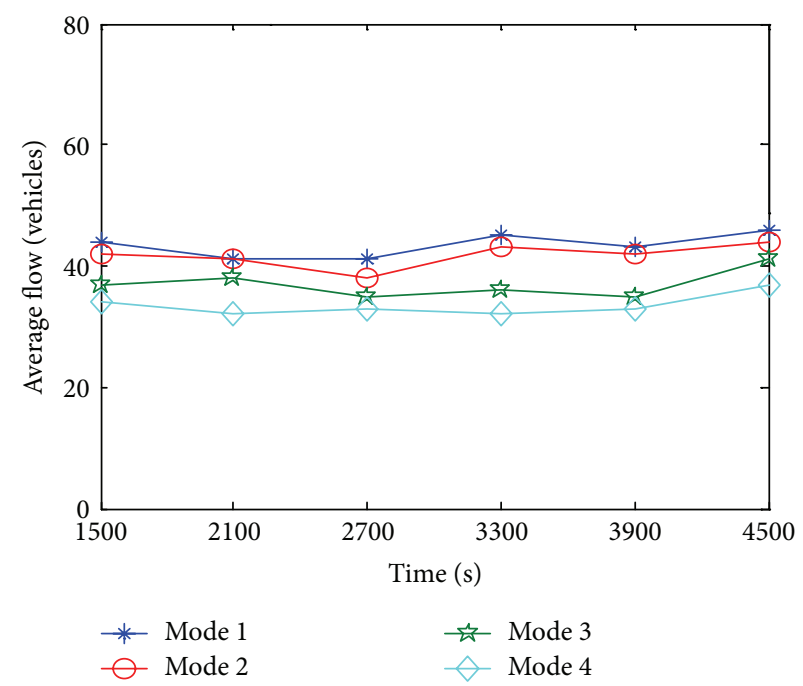

(a)

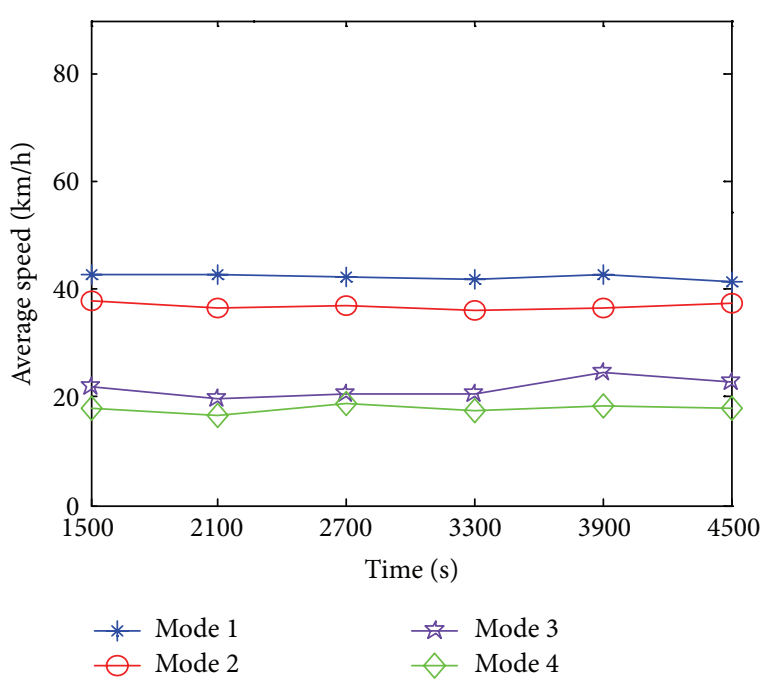

(b)

FIGURE 6: Average flow and average speed in every $600 \mathrm{~s} \mathrm{time} \mathrm{interval} \mathrm{for} \mathrm{each} \mathrm{behavior} \mathrm{mode} \mathrm{((a)} \mathrm{average} \mathrm{flow} \mathrm{versus} \mathrm{time} \mathrm{curves;} \mathrm{(b)} \mathrm{average}$ speed versus time curves).

TABLE 3: Statistical results for the left-turning traffic flow.

Simulation-based flow (no conflict, $P_{1}=1.0, \quad$ Simulation-based flow $\left(P_{1}=0.5, P_{2}=0.1\right.$, $\left.P_{2}=P_{3}=P_{4}=0\right)(\mathrm{veh} / \mathrm{h})$ $\left.P_{3}=0.3, P_{4}=0.1\right)(\mathrm{veh} / \mathrm{h})$

Field-data-based flow (veh/h)

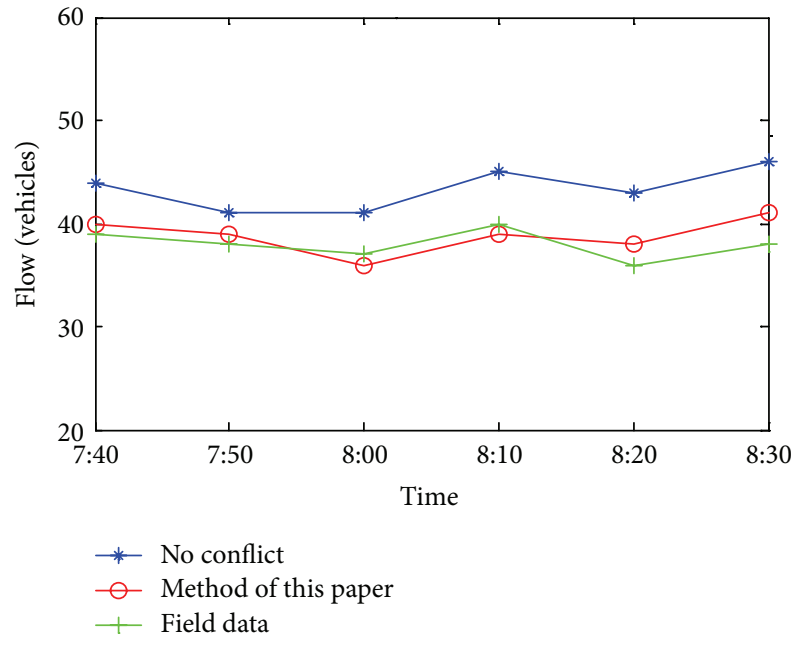

FIGURE 7: Detailed statistical results of left-turn traffic flow in each fixed time interval.

mixed traffic conditions in developing countries. These models and methods proposed in this paper provide an important theoretical basis and a methodology for modeling left-turn driving behaviors at signalized intersections with mixed traffic condition. The results will be of significant benefit for the planning and design of mixed traffic intersections to reduce the probability of pedestrian violations and to improve intersection capacity and safety. By simulating four basic driving behavior modes, we obtained both the microscopic characteristics of driving behaviors (instantaneous velocities, locations, and headway allowances of individual vehicles) and the macroscopic parameters of traffic flow (average flow, density, and speed) in the affected region. Mode 1 has better evaluation outputs in terms of delay time and passing efficiency with mixed traffic conditions than other modes. The driving behaviors of Modes 2, 3, and 4 should be reduced in field intersections, especially for mixed traffic conditions. The simulation results revealed the impact of different degrees of vehicle-pedestrian conflict on left-turn driving behavior at signalized intersections, hence, can be referred for practical engineering applications of intersection design. To analyze the relative share of each behavior mode and to verify the validity of the simulation models proposed in this paper, we compared the results of our simulations with the field data for the simulated intersection based on the proposed logit model. The determination of the relative share will be useful for traffic simulation applications on signalized intersections with mixed traffic conditions. Enhancing the ability to control violations in traffic engineering issues will efficiently improve intersection capacity and safety.

Future studies may focus on combined behavior modes of left-turning traffic, which can better reflect the mixed traffic conditions encountered in real-world scenarios. Moreover, the driving behavior modes will be more complex at unsignalized intersections under mixed traffic conditions, and, thus, there is a need for more general models and more detailed field data to simulate, compare, and analyze the behaviors observed in such cases. 


\section{Competing Interests}

The authors declare that there are no competing interests regarding the publication of this paper.

\section{Acknowledgments}

This work was supported by the China Postdoctoral Science Foundation (2015M570912) and the Open Fund for a Key-Key Discipline of Zhejiang University of Technology (2015001). Support from the International Postdoctoral Exchange Fellowship Program (2015037) is also gratefully acknowledged.

\section{References}

[1] P. Abishai, "Driver behaviour and accident records at unsignalized urban intersections," Accident Analysis \& Prevention, vol. 17, no. 1, pp. 25-32, 1985.

[2] P. Bonsall, R. Liu, and W. Young, "Modelling safety-related driving behaviour-impact of parameter values," Transportation Research Part A: Policy and Practice, vol. 39, no. 5, pp. 425444, 2005.

[3] G. M. Björklund and L. Åberg, "Driver behaviour in intersections: formal and informal traffic rules," Transportation Research Part F: Traffic Psychology and Behaviour, vol. 8, no. 3, pp. 239-253, 2005.

[4] X. Yan and E. Radwan, "Effect of restricted sight distances on driver behaviors during unprotected left-turn phase at signalized intersections," Transportation Research Part F: Traffic Psychology and Behaviour, vol. 10, no. 4, pp. 330-344, 2007.

[5] J. Zhang, K. Suto, and A. Fujiwara, "Effects of in-vehicle warning information on drivers' decelerating and accelerating behaviors near an arch-shaped intersection," Accident Analysis \& Prevention, vol. 41, no. 5, pp. 948-958, 2009.

[6] T. Limanond, P. Prabjabok, and K. Tippayawong, "Exploring impacts of countdown timers on traffic operations and driver behavior at a signalized intersection in Bangkok," Transport Policy, vol. 17, no. 6, pp. 420-427, 2010.

[7] M. Elhenawy, A. Jahangiri, H. A. Rakha, and I. El-Shawarby, "Modeling driver stop/run behavior at the onset of a yellow indication considering driver run tendency and roadway surface conditions," Accident Analysis and Prevention, vol. 83, pp. 90-100, 2015.

[8] H. Bar-Gera, O. Musicant, E. Schechtman, and T. Ze'evi, “Quantifying the yellow signal driver behavior based on naturalistic data from digital enforcement cameras," Accident Analysis \& Prevention, 2015.

[9] D. Lin, W. Ma, L. Li, and Y. Wang, "A driving force model for non-strict priority crossing behaviors of right-turn drivers," Transportation Research Part B: Methodological, vol. 83, pp. 230244, 2016.

[10] E. Muhrer and M. Vollrath, "Expectations while car followingthe consequences for driving behaviour in a simulated driving task," Accident Analysis and Prevention, vol. 42, no. 6, pp. 21582164, 2010.

[11] R. Riccardo, G. Massimiliano, G. Gregorio, and M. Claudio, "Comparative analysis of random utility models and fuzzy logic models for representing gap-acceptance behavior using data from driving simulator experiments," Procedia-Social and Behavioral Sciences, vol. 54, pp. 834-844, 2012.
[12] F. Rosey and J.-M. Auberlet, "Driving simulator configuration impacts drivers' behavior and control performance: an example with studies of a rural intersection," Transportation Research Part F: Traffic Psychology and Behaviour, vol. 27, pp. 99-111, 2014.

[13] Y. Matsumoto and G. Peng, "Analysis of driving behavior with information for passing through signalized intersection by driving simulator," Transportation Research Procedia, vol. 10, pp. 103-112, 2015.

[14] A. Habibovic, E. Tivesten, N. Uchida, J. Bärgman, and M. L. Aust, "Driver behavior in car-to-pedestrian incidents: an application of the Driving Reliability and Error Analysis Method (DREAM)," Accident Analysis \& Prevention, vol. 50, pp. 554$565,2013$.

[15] I. A. Kaysi and A. S. Abbany, "Modeling aggressive driver behavior at unsignalized intersections," Accident Analysis \& Prevention, vol. 39, no. 4, pp. 671-678, 2007.

[16] S. H. Hamdar, H. S. Mahmassani, and R. B. Chen, "Aggressiveness propensity index for driving behavior at signalized intersections," Accident Analysis and Prevention, vol. 40, no. 1, pp. 315-326, 2008.

[17] M. Danaf, M. Abou-Zeid, and I. Kaysi, "Modeling anger and aggressive driving behavior in a dynamic choice-latent variable model," Accident Analysis \& Prevention, vol. 75, pp. 105-118, 2015.

[18] C.-K. Chen, J. Li, and D. Zhang, "Study on evacuation behaviors at a T-shaped intersection by a force-driving cellular automata model," Physica A: Statistical Mechanics and Its Applications, vol. 391, no. 7, pp. 2408-2420, 2012.

[19] X. Hu, W. Wang, and H. Yang, "Mixed traffic flow model considering illegal lane-changing behavior: simulations in the framework of Kerner's three-phase theory," Physica A: Statistical Mechanics and its Applications, vol. 391, no. 21, pp. 5102-5111, 2012.

[20] G. Chen, F. Meng, G. Fu, M. Deng, and L. Li, "A cell automation traffic flow model for mixed traffic," Procedia-Social and Behavioral Sciences, vol. 96, pp. 1412-1419, 2013.

[21] X. Liang, Z.-L. Liu, and K. Qian, "Capacity analysis of signalized intersections under mixed traffic conditions," Journal of Transportation Systems Engineering and Information Technology, vol. 11, no. 2, pp. 91-99, 2011.

[22] J. Prasetijo and H. Ahmad, "Capacity analysis of unsignalized intersection under mixed traffic conditions," Procedia-Social and Behavioral Sciences, vol. 43, pp. 135-147, 2012.

[23] J. Prasetijo, W. Razzaly, N. Wu et al., "Capacity analysis of priority intersections with flare under mixed traffic conditions," Procedia-Social and Behavioral Sciences, vol. 138, pp. 660-670, 2014.

[24] S. Marisamynathan and P. Vedagiri, "Modeling pedestrian delay at signalized intersection crosswalks under mixed traffic condition," Procedia-Social and Behavioral Sciences, vol. 104, pp. 708-717, 2013.

[25] S. Anjana and M. V. L. R. Anjaneyulu, "Safety analysis of urban signalized intersections under mixed traffic," Journal of Safety Research, vol. 52, pp. 9-14, 2015.

[26] R. Wiedemann, Simulation des Straßenverkehrsflusses, vol. 8 of Schriftenreihe Heft, Instituts für Verkehrswesen der Universität Karlsruhe, Karlsruhe, Germany, 1974.

[27] R. Wiedemann, "Modelling of RTI-elements on multi-lane roads," in Drive Conference, vol. 2, Brussels, Belgium, 1991.

[28] R. Wiedemann and U. Reiter, "Microscopic traffic simulation: the simulation system MISSION, background and actual state," 
CEC Project ICARUS (V1052), Final Report 2, Appendix A., Brussels, Belgium, 1992.

[29] R. E. Campbell and L. E. King, "The traffic conflicts technique applied to rural intersections," Accident Analysis \& Prevention, vol. 2, no. 3, pp. 209-221, 1970.

[30] X. Liu and H. L. Duan, "Research on standard program of traffic conflict techniques at intersections," Technology of Highway and Transport, vol. 14, no. 3, pp. 29-34, 1997.

[31] B. J. Schroeder, A behavior-based methodology for evaluating pedestrian-vehicle interaction at crosswalks [Ph.D. dissertation], North Carolina State University, 2008.

[32] C. E. Shannon, "Von Neumann's contributions to automata theory," Bulletin of the American Mathematical Society, vol. 64, pp. 123-129, 1958.

[33] S. Wolfram, Theory and Applications of Cellular Automata, vol. 1, World Scientific, Singapore, 1986.

[34] K. Nagel and M. Schreckenberg, "A cellular automaton model for freeway traffic," Journal de Physique I, vol. 2, no. 12, pp. 22212229, 1992.

[35] Biogeme, http://biogeme.epfl.ch/home.html. 


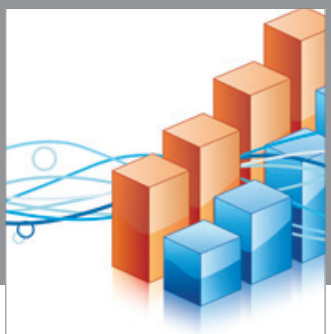

Advances in

Operations Research

vatem alat4

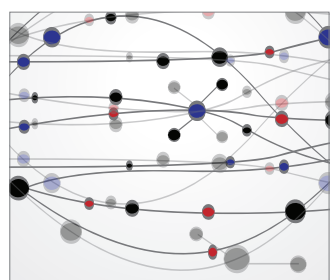

\section{The Scientific} World Journal
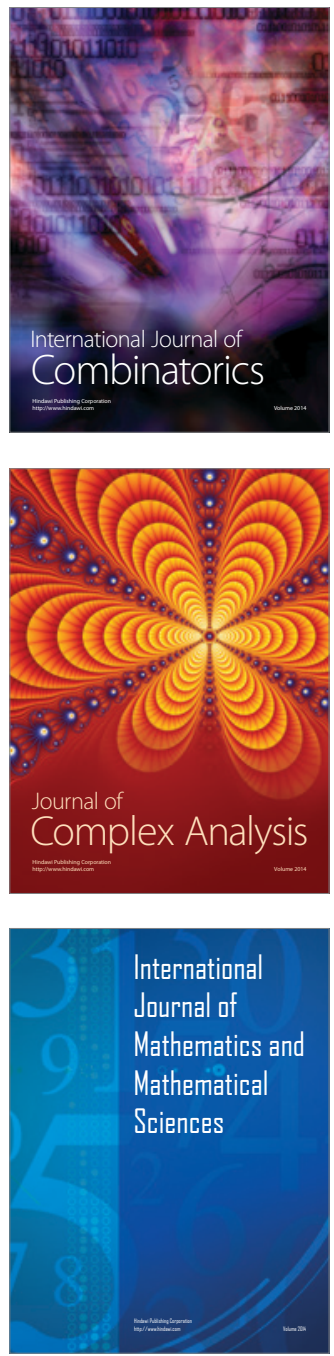
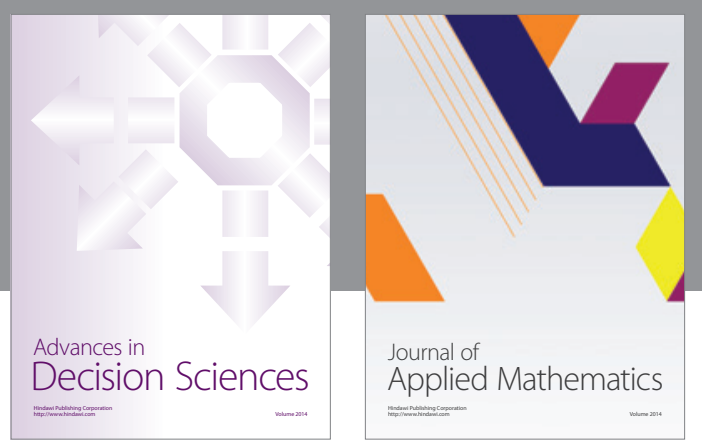

Algebra

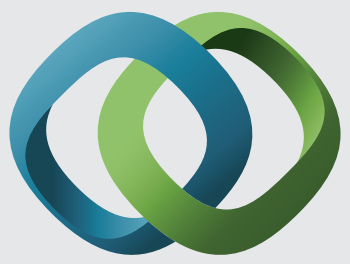

\section{Hindawi}

Submit your manuscripts at

http://www.hindawi.com
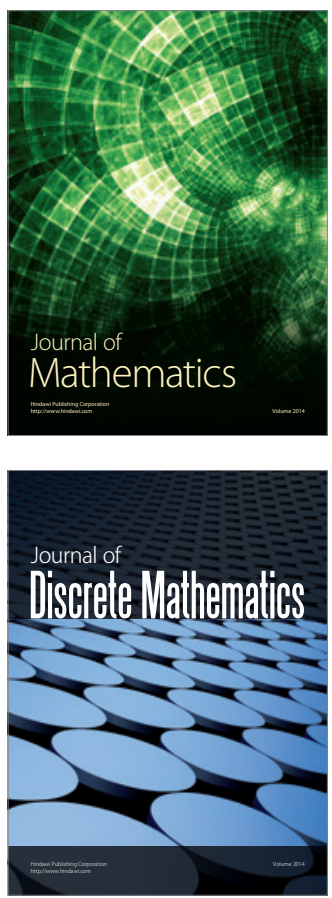

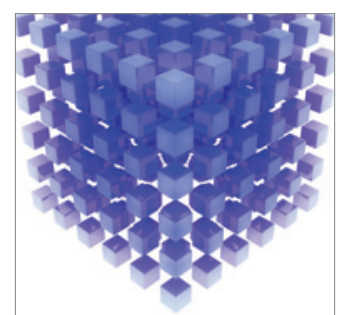

Mathematical Problems in Engineering
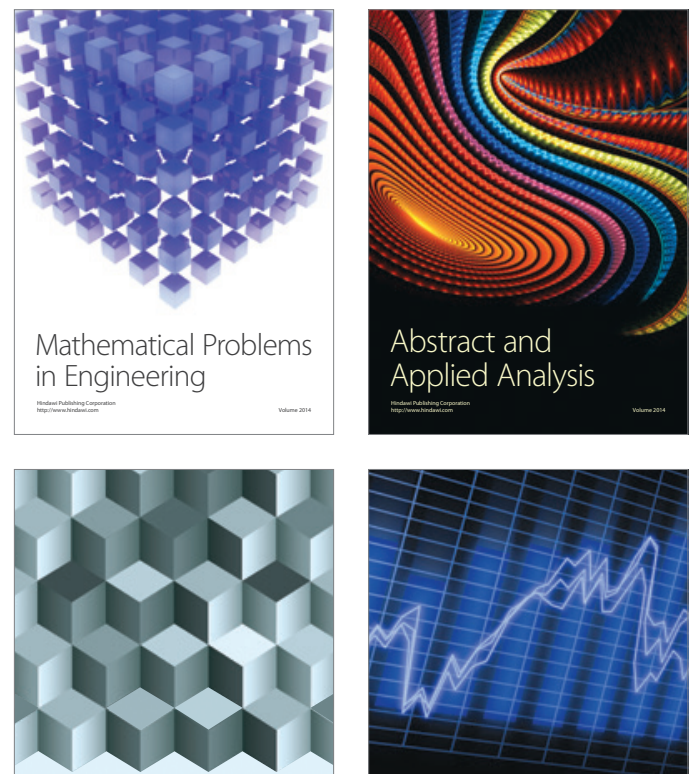

Journal of

Function Spaces

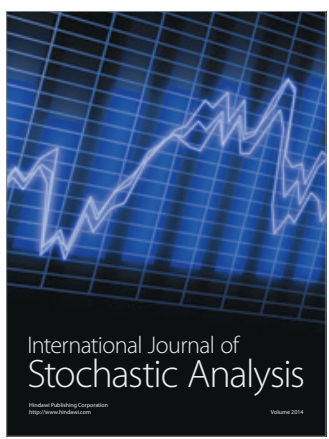

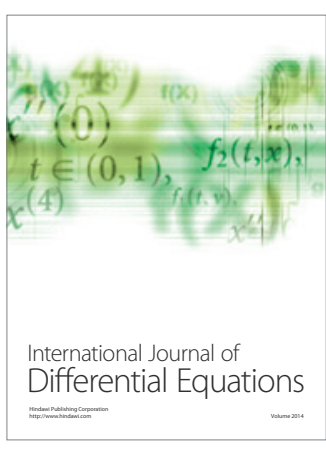
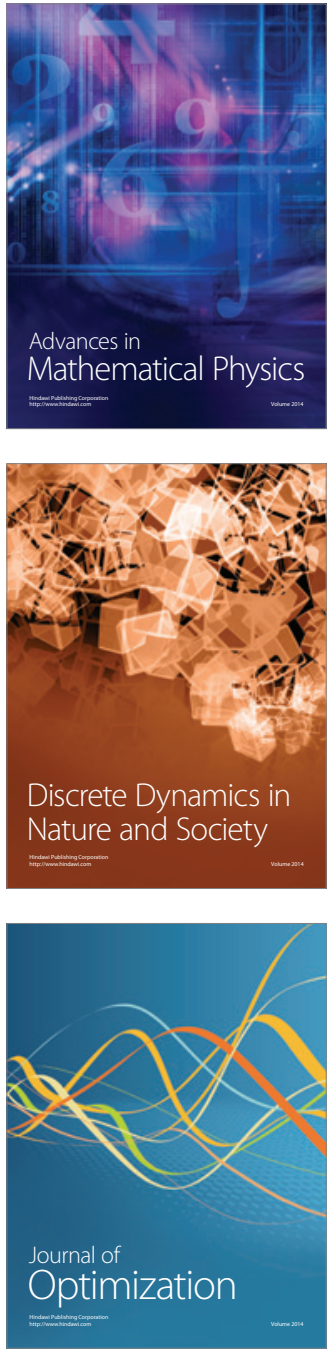